\title{
Dynamic Organizational Capabilities: The Joint Effect of Agility, Resilience and Empowerment
}

\author{
Mahmoud Abdelaziz Elgamal \\ Management and Marketing Department, Kuwait University, Kuwait
}

Email address:

elgamal8@yahoo.com

To cite this article:

Mahmoud Abdelaziz Elgamal. Dynamic Organizational Capabilities: The Joint Effect of Agility, Resilience and Empowerment. Journal of Human Resource Management. Vol. 6, No. 2, 2018, pp. 44-49. doi: 10.11648/j.jhrm.20180602.11

Received: May 15, 2018; Accepted: June 14, 2018; Published: July 10, 2018

\begin{abstract}
The paper presents a model of Dynamic Organizational Capabilities (ODCs). The capabilities included are organizational agility, Organizational resilience and human resources empowerment. These capabilities were selected based on reviews of the literature, which showed the potency of these capabilities in terms of their single and joint impact over organizational vitality (OV). The paper went beyond providing a conceptualization of ODCs, to provide an empirical test of it. The model was tested using regression analysis. The results show that validity of the proposed ODC model. The organizational resilience acted as a moderator of the relationship between organization agility and $\mathrm{OV}$. Thus the organizational resilience interaction with organization agility improved significantly the variance explained in OV. On the other hand human resources empowerment acted as a mediator between organizational agility and OV. The conceptual and empirical implications of the results are discussed and the limitations of the study noted, future research direction were pointed at.
\end{abstract}

Keywords: Dynamic Organization Capabilities, Organizational Agility, Organizational Resilience, Human Resources Empowerment, Organizational Vitality

\section{Introduction}

The current study is following the lead of several scholars who introduced the conceptualization of Dynamic Managerial capabilities [19]. Specifically this study is introducing the conceptualization of Dynamic Organizational Capabilities (ODCs). These capabilities explain how organizations can survive and sustain competitive advantages by responding to environmental change, and by creating favorable environmental changes [19]. The domain of DOCs is wide and complex. It covers strategic issues, organization vision, organization design, interactions with the environment and environmental change.

There are different versions of ODCs [19]. Sune and Gibb introduced a set of ODCs including adding, transferring, integrating and shedding and two higher - order capability, goal development and change orchestration [18]. The current study advances a specific set of ODCs, that includes organizational agility, organizational resilience and human resources empowerment. Reviews of the literature indicate that the above named variables are dominant in the main literature streams $([5,12,1])$.

\section{Literature Review}

The review of the literature indicate that the work in the area of ODCs remain mostly conceptual and focusing on the foundational level of analysis, and that there is scarcity in the empirical work reported in the literature [19]. The literature is characterized as being theoretical rather than empirical. And most of the studies introduced one capability and theorized about its impact over the organization performance.

Many studies explored the construct of organizational agility and proposed a positive impact over OV (i.e. ([1, 3, 9]). Other stream of research focused on organizational resilience (i.e. [12-17]). Yet some other research focused on other ODCs, for example Goodman introduced engagement as an important variable for organizational vitality [8]. Many studies examined the impact of human resources empowerment (i.e. $[20,11])$.

Other studies explored the interaction of agility, resilience and green. This was explored conceptually. Alhadid [1] examined the Joint effect of resilience capacity and strategic agility and on organization thriving. The examination was at 
the conceptual level. Carvalho [6] examined the simultaneous deployment of agile and resilient approaches impact over the supply chain performance and competitiveness using a conceptual level.

Yet very few empirical studies reported, notably the study Sure and Gibb [18]. Which explored the pattern of organizational change empirically. The current study advances conceptualizations of ODCs that include three very frequently reported variables; namely organizational agility, organization resilience and human resources empowerment. The current study goes beyond introducing a conceptualization of ODCs to testing empirically the proposed model.

\section{Data and Methodology}

\subsection{Objectives}

The study has two fold objectives as follows:

i. To advance a specific set of ODCs, based on extensive review of the literature. The set includes organizational agility, organizational resilience and human resources empowerment the dependent variable is organization vitality $(\mathrm{OV})$.

ii. To test the validity of the proposed ODCs in terms of their Join impact over OV.

\subsection{The Study Model}

The following figure (Figure 1) depicts the study model

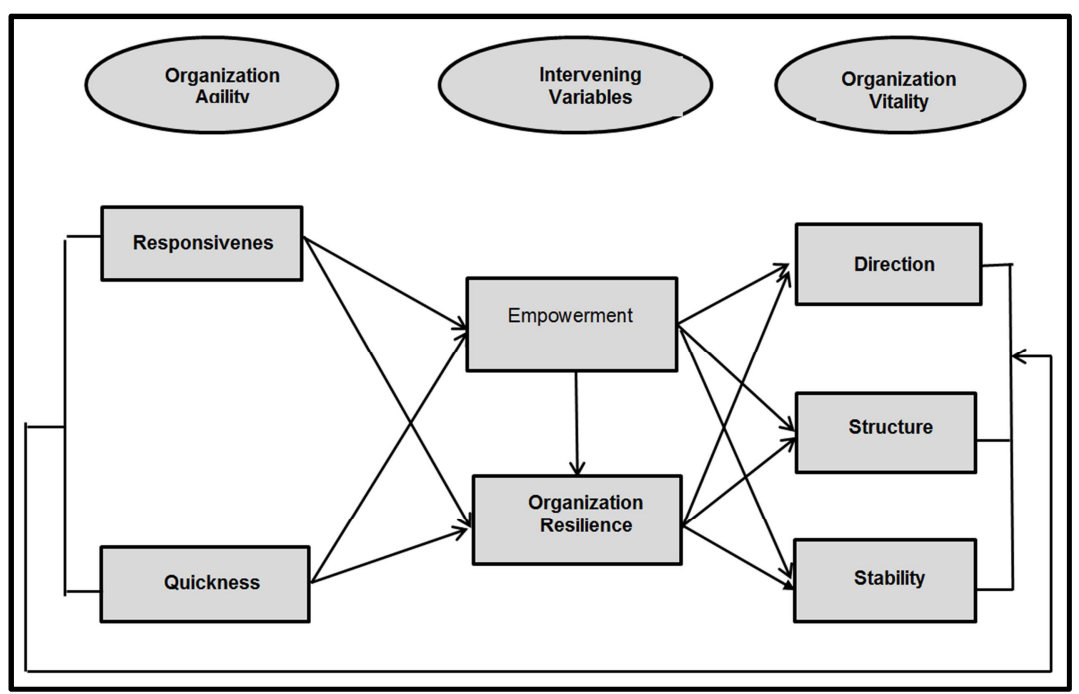

Figure 1. The Study Model.

The definitions of the Model variables are as follows

Organizational Agility

Agility is the ability of an organization to renew itself, adapt, change quickly and succeed in a rapidly changing, ambiguous, turbulent environment ([3, 14, 9, 6]).

Organization Resilience

An organization's resilience captures its ability to take situation - specific robust, transformative actions when confronted with unexpected and powerful events that have the potential to jeopardize an organization's long term survival [7, $14,17])$. It is worth noting that resilience is distinguished from agility; because it deals with powerful, significant changes that threatens the organizations life $([11,13,20])$.

\section{Empowerment}

Empowerment is a pillar that describes the relationship between leadership and employees through, authority, autonomy and other factors. It refers to the degree with which the powers of organizational leaders and lower - level employees are distributed, separated or shared $([11,13,20])$.

\section{Organizational Vitality $(\mathrm{OV})$}

$\mathrm{OV}$ is defined as the degree to which an organization has continually been successful in meeting performance expectation in the eyes of its customer, employees, stakeholders, and relevant communities, which results in the organization remaining viable and stable overtime ([4, 8, 17, 22]). The OV has three major dimensions as follows

\section{Structure}

Structure measures the strength of the systems that are currently in place, if policies and procedures line up with organizational needs, and if departmental expectations and job duties are clear. People need to know what is expected, what their role in the organization is, and just how the organization is managed.

\section{Direction}

Direction focuses on the strategy and vision that the leadership of an organization creates and promotes throughout the organization. People need to have a clear roadmap of the future of their organization and understand how they will reach significant milestones. Direction is strengthened when there is a unified strategy and vision that all staff are connected and committed to carrying.

\section{Stability}

Stability zeros in on an organization's ability to attract, hire, and retain top talent. It also gives a clear picture of how effectively the organization comes together when challenges arise, if change is managed effectively, and whether or not 
employees feel the organization provides adequate support to truly be successful. People need to know that they are not on a "sinking ship" and that they have what it takes to succeed.

It is to be noted that the study model above, proposes a direct impact of organizational agility on $\mathrm{OV}$, as well as indirect impact. The latter is through the impact of the interaction between organizational agility and organizational resilience and the interaction between organizational agility and empowerment on $\mathrm{OV}$.

\subsection{Hypotheses}

The study advances three hypotheses:

i. The Join effect of organizational agility and organizational resilience has significant impact over OV.

ii. The Joint effect of organizational agility and human resources empowerment has significant impact over OV.

iii. The Joint effect of organizational agility and organizational resilience and human resources empowerment has significant impact over OV.

\subsection{Data}

After developing the proposed conceptual model based on the literature reviews, the stage was set to empirically test the proposed model. Data were collected using a seven point likert type scale questionnaire. The subjects were professionals and human resources staff working in 19 Kuwaiti organizations. These organizations are operating in the fields of financial services and IT. The number of respondents is 177 subjects. They were select based on their tenure that is the subjects have to have minimum two years experience in their respective organizations. This condition was set to ensure the familiaityr of the subjects within their respecedt organizations.

\section{Results}

The descriptive statistics and reliability analysis of Cronbach Alpha are depicted in table 1. The reliability analysis indicates that Cronbach Alpha for the study variables ranged from .78 to .96 , which shows acceptable levels of reliabilities.

Table 1. Descriptive Statistics \& Reliability Analysis.

\begin{tabular}{lllll}
\hline Variables & N & Mean & $\begin{array}{l}\text { STD } \\
\text { Deviation }\end{array}$ & $\begin{array}{l}\text { Cronback } \\
\text { Alpha }\end{array}$ \\
\hline Responsiveness & 177 & 4.78 & 1.18 & .88 \\
Quickness & 176 & 4.43 & 1.15 & .88 \\
Organization Resilience & 177 & 5.74 & .97 & .89 \\
Empowerment & 177 & 5.52 & .86 & .78 \\
Direction & 177 & 4.61 & 1.43 & .91 \\
Structure & 174 & 5.00 & 1.19 & .96 \\
Stability & 177 & 5.27 & 1.30 & .82 \\
\hline
\end{tabular}

The results of the regression analysis will be discussed, under the three following subheadings:

1) The interaction between organizational agility and organizational resilience.

2) The interaction between organizational agility and human resources empowerment.

3) The interaction among organizational agility, organizational resilience and human resources empowerment.

1. Regression analysis results of the interaction between organizational agility and organizational resilience.

The results of regression analysis in terms of the variance explained of $\mathrm{OV}$ is depicted in figure 2. As the values of the adjusted R-square show, the interaction between organizational agility and organizational resilience. The interaction effect improved significantly the values of the variance explained.

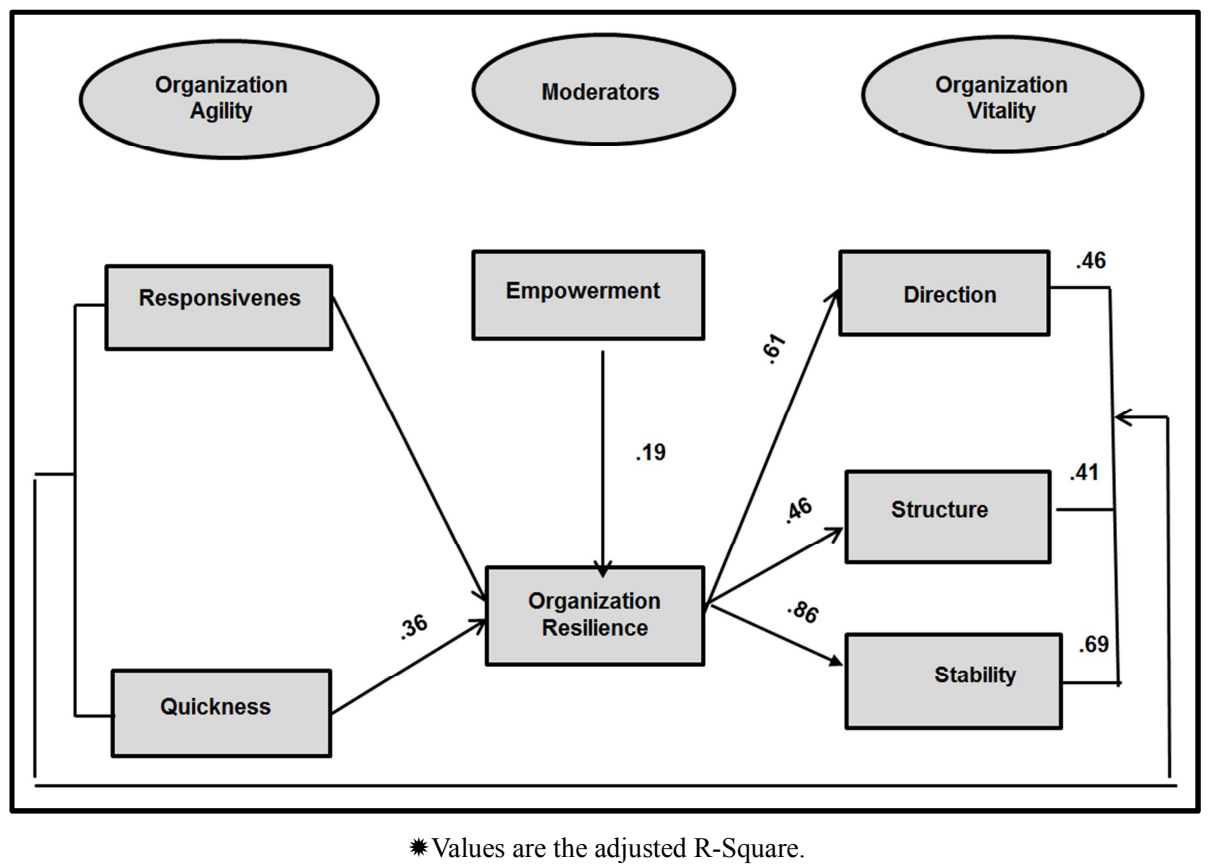

Figure 2. The summary of Regression Analysis Results. The impact of organizational agility on organization vitality \& The Joint impact of organizational agility and organization resilience on Organization vitality. 
The following Table (Table 2) depicts the values of Betas for the three OV dimensions

Table 2. The Impact of the Interaction of Organization Agility and Resilience on Organization Vitality.

\begin{tabular}{lllllll}
\hline Independent & Direction & \multicolumn{2}{l}{ Structure } & \multicolumn{2}{l}{ Stability } \\
\cline { 2 - 7 } Variables & Standardized Coefficients Beta & Sig & Standardized Coefficients Beta & Sig & Standardized Coefficients Beta & Sig \\
\hline Constant & ---- & .0001 & & .002 & & .0001 \\
Agility & -.098 & .166 & .21 & .03 & .13 & .001 \\
Resilience & -.73 & .0001 & -.01 & .88 & -.40 & .0001 \\
Agility** Resilience & 1.48 & .0001 & .52 & .001 & 1.17 & .0001 \\
Adjusted & .73 & & .47 & & .92 & \\
\hline
\end{tabular}

The above table shows that there is a significant interaction between organizational agility and resilience, where all the Betas of the interactions are significant. Specifically, it was found that organizational resilience acts as a moderator between agility and OV. Where a moderator variable is defined as a third variable that affects the strength of the relationship between an independent and dependent variables (Hayes, 2013). The interaction is shown to improve the values of the variance explained significantly in OV dimension. Namely for the direction the variance explained by the organizational agility (the adjusted R-square) is .46, while the interaction between organizational agility and organizational resilience explained .61 of the variance. The same is True of the OV dimension of structure. The variance explained by the organizational agility is .41 , while the interaction between organizational agility and organizational resilience explained .46 . As of the OV dimension of stability it was also true. The variance explained by the organizational agility is .69 while the interaction between organizational agility and organizational resilience explained .86 of the variance.

The above results lend support to the hypothesis 1 . That states the joint effect of organizational agility and organizational resilience has significant impact over OV.

2. Regression analysis results of the interaction between organizational agility and human resources empowerment. The result of the regression analysis in terms of the variance explained in OV is depicted in figure 3 As the values of the adjusted R-square shows, the interaction between organizational agility and human resources did not improve the variance explained in OV. The interaction did not improve the variance explained in $\mathrm{OV}$ for any of the $\mathrm{OV}$ dimensions

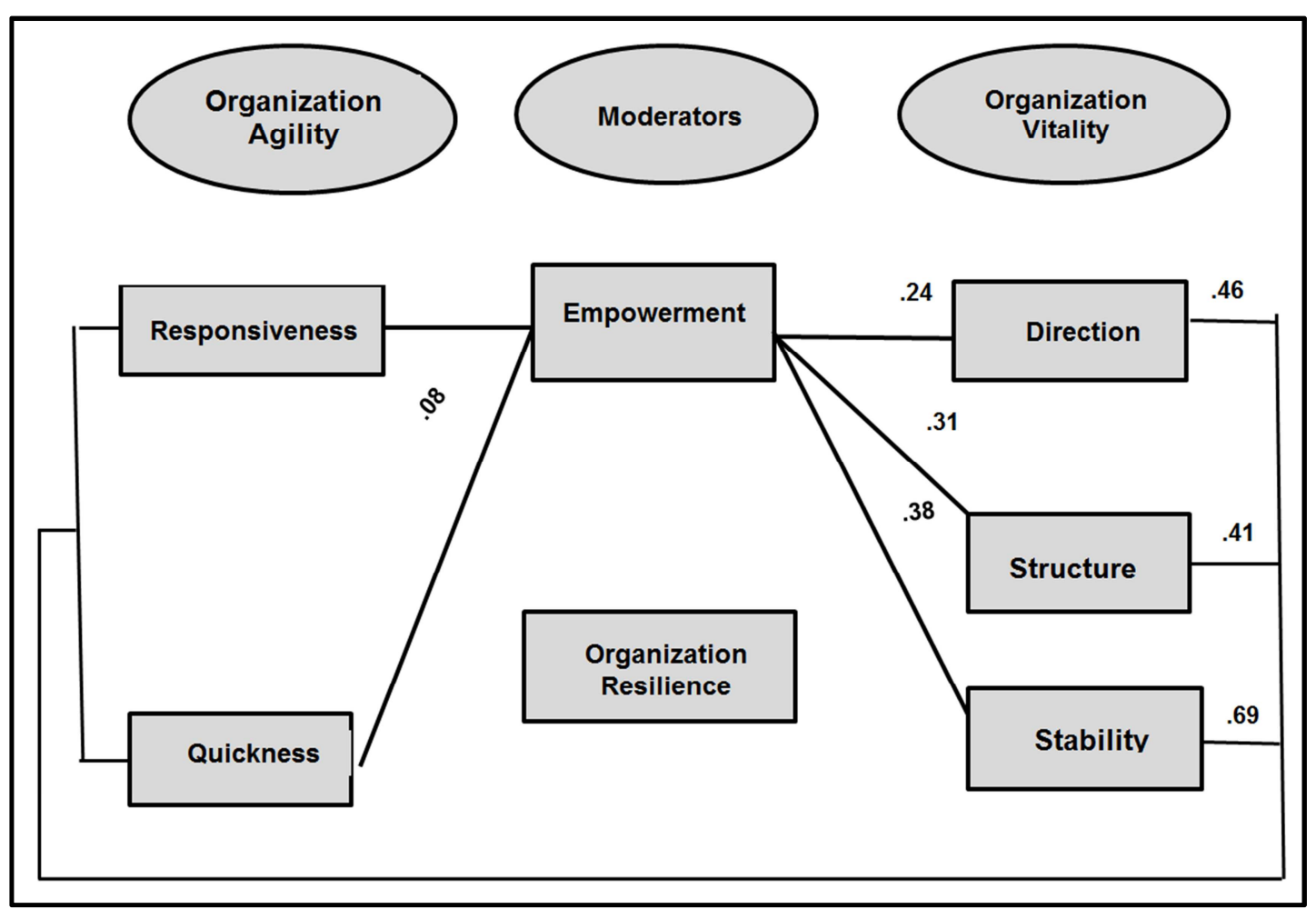

*Values are the adjusted R-Square.

Figure 3. The summary of Regression Analysis Results. The impact of organizational agility on organization vitality \& The Joint impact of organizational agility and Empowerment on Organization vitality.

The following Table (Table 3) depicts the values of Betas for the three OV dimensions 
Table 3. The Impact of the Interaction of Organization Agility and Resilience on Organization Vitality.

\begin{tabular}{lllllll}
\hline $\begin{array}{l}\text { Independent } \\
\text { Variables }\end{array}$ & Direction & & Structure & \multicolumn{2}{l}{ Stability } \\
\cline { 2 - 7 } & Standardized Coefficients Beta & Sig & Standardized Coefficients Beta & Sig & Standardized Coefficients Beta & Sig \\
\hline Constant & & & & .01 & & .001 \\
Agility & .58 & .0001 & .45 & .0001 & .65 & .0001 \\
Resilience & .00 & .998 & -.09 & .33 & -.26 & .0001 \\
Agility**Empowerment & .18 & .16 & .40 & .001 & .49 & .0001 \\
Adjusted & .48 & & .47 & & .75 & \\
\hline
\end{tabular}

The above Table shows that there is significant interaction between organizational agility and human resources empowerment for two of the Betas namely for the $\mathrm{OV}$ dimensions of structure and stability specifically it was found that the human resources empowerment acts as a mediator between organizational agility and OV. The mediator is defined as a variable that affects another variable, which in turn affects the dependent variable (Hayes, 2013). That is human resources empowerment contributes to organizational agility, which in turn contributes to OV.

The above results lend support to the hypothesis 2 . That states the joint

Effect of organizational agility and human resources empowerment has significant impact over OV.

The study did not include the results of the Joint interaction of organizational agility, organizational resilience and human resources empowerment, simply because of the fact that resilience and empowerment are having different relationships with organizational agility, the first acts as a moderator and the second acts as a mediator.

\section{Discussion}

The conceptualization of the DOCs, seem to be very promising since it groups together related important variables to improve the predictability of $\mathrm{OV}$. The study attempted to go beyond advancing a conceptualization of ODCs, by providing an empirical test for it which is very required research effort [18]. The review of the literature indicate that agility is an ongoing processes. It is an evolving phenomenon, and it is similar in that respect to continuous improvement $([2,21])$.

Organizational agility is fundamentally related to quickness and responsiveness to dealing with environmental changes.

However organizational resilience is related to dealing to environmental sudden and significant disruption in order to survive and prosper. Resilience reaches far beyond risk management toward having a comprehensive view of and capabilities to maintain the organization health and success.

The pillars of human resources empowerment are related to trusting qualified and highly competent human resources, who have the decision making authority to make proper and quick responses within their respective domain of responsibility.

The study has conceptual and practical imprecations. On the conceptual side the implications. It seems that The ODCs conceptualizations help in grouping important variables together that interact and improve the predictability and understanding of the dependent variable. In addition the interactions among the dimension of ODCs may have different types. For instance in the current study, it was found that variables may act as moderators, or mediators. One can say also that The interaction between/ among variables of ODCs may have differential impact on the dimensions of the dependent variable.

The practical implications It is suggested that Organizations should strive to build a multi-dimensional culture that accepts different but related variables such as agility, resilience, and empowerment. As well Organizations should build resilience capabilities to survive in the very turbulent and surprising environment. It is also suggested that

Organizations should make human resources empowerment a deep rooted practice.

The limitations of the study include using perceptual data only and method to measure the independent and dependent variable. So it is subject to are method bias. The future research should Use hard data to measure organization vitality. And should also include other ODCs in addition to the ones used in the current study such as engagement.

\section{Conclusion}

The study provided some evidence of the conceptualization of ODCs; which is based on having a number of constructs that are related, reinforcing and complementary in terms of explaining OV. The focus of the ODCs conceptualization is on the interactions. In the real organizational life the main concern is focused on the interactions among variables rather than the single effects of variables.

It was shown in the study that it is imperative to recognize the multidimensionality of the dependent which is OV. The ODCs had differential impact on the different dimensions of $\mathrm{OV}$.

It is evident that the introduction of conceptualizations that seem compelling is not as useful as empirically testing the conceptualization. The testing of the ODCs conceptualization introduced in the study shed light on the complexity of the relationships among the ODCs. The resilience acted as a moderator for the relationship between agility and OV; while human resources empowerment acted as a moderator of the relationship between agility and $\mathrm{OV}$.

The results of the study suggest that organizations should tackle the challenge of creating and adopting a multidimensional organizational culture that support simultaneously agility, resilience and human resources empowerment. 


\section{References}

[1] Alhadid Anasy. (2016). The Effect of Organization Agility on Organization Performance International Review of Management Business Research, Volume 5, Issue 1.

[2] Alzoobi, A. E., Al-otoun, F. J. \& Albatainh. (2011). Factors Associated Affecting Organization Agility on Product Development.. International Journal of Research and Reviews in Applied Sciences, Volume 9.

[3] Ashton, Gary. (2015). The keys to Organizational Agility. http://www.management-issues.com/opinion/6116/The-keysto organizational agility/

[4] Bishwas, Sunant Kumar. (2014). Critical Processes for Organization Vitality. A Conceptual study. http:// link.springer.com/chapter/ 10.1007/978-81-322-2151-7

[5] Carvalho, H. Azevedo Susana garrido \& Cruz-Machado. (2012). Agile and Resilience Approach to supply chain Management: Influence on Performance and Competitiveness. Logistics Research, Volume 4, Issue 1-2.

[6] Carvalho, Helena \& Durate, Susana. (2011). Lean, Agilie, Resilient and green: Divegencie and Synergies. http://www.emeralcinsight.com/doi/full/10.1108/2040146\111 135037.

[7] Everly, George S. (2011). Building Resilient Organizational Culture. HBR.

[8] Goodman Ricky (2016). Employee Engagement or Organizational Vitality. Http:// www. LinkedIn. Com/pulse/ employee-engagement- organizational/-vitality-RickyGoodman.

[9] Harraf Abe, Wanasika, Isaac, tate kaylynn \& Talbott, Kaitly. (2015). Organizational Agility. The Journal of Applied Research- March/April, Volume 31, issue 2.

[10] Hayes, A, F. (2013). Introduction to Mediation, Moderation and conditional process Analysis: A Regression -Based Approach, New York, NY, Guilford Press.

[11] Healthfield, Susan M. (2017). Top principles of Employee
Empowerment. http://www. Thebalance.com/top-principlesof- employee -empowerment- 1918658.

[12] Helfat constance E. \& Peteraf, Margaret, A (2009). Understanding Dynamic Capabilities: progress Along a Developmental Path Strategic Organization, Volume 9, issue 1.

[13] Kerr Howard. (2016). Analysis Organizational Resilience Harnessing Experience, Embracing Opportunity, Quality magazine, July.

[14] Lengnick -Hall, Cynthia A. Beck Tammy E. (2009). Resilience Capacity and Strategic Agility: Prerequisites for Thriving in a Dynamic Environment. The University of Texas at San Antonio, College of Business. Working paper series.

[15] National Academy of Sciences. (2012). Understanding Individual and Organizational Resilience and Performance Measures. http://www. Ncbi.hlm.nih.gov/books/ NBK201581/

[16] Okotoh, Argwings K. (2015) Influence of Organizational Agility on Operational performance of Trademark East Africa. A Master Thesis, University of Nairobi.

[17] Seville, Erica. (2016). What makes a Resilient Organization. http:// www. Koganpage.com/article/what- makes-a- resilientorganization.

[18] Sune Albert Gibb Jenny. (2015). Dynamic Capabilities as patterns of Organizational change. An empirical study on transforming a firm's Resource Base. http:// www.Emerldinsight.com/doi/full/10.1108/JOCM-01-20150019 .

[19] Teece, David J. (2007). Dynamic Capabilities. http:// en. Wikipedia.org/wiki/David_Teece

[20] Tohidi. Hamid. (2011). The Aspects of Empowerment of Human Resources. Procedic Social and Behavioral Science, Volume 31.

[21] Williams T. Worley C. G \& Lawler 111, E. E. (2013). The Agility factor strategy Business. April.

[22] Xenidis, Yiannis \& Treocharous, kyriakos. (2014). Organizational Health: Definition and Assessment. http:// doi.org/10.1016/J. proeng.2014/10.584. 